\title{
Emulating prosthetic feet during the prescription process to improve outcomes and justifications
}

\author{
(Invited Presentation)
}

\author{
Joshua M. Caputo \\ Dept. Mechanical Engineering \\ Carnegie Mellon University \\ Pittsburgh, Pennsylvania 15213 \\ jmcaputo@andrew.cmu.edu
}

\author{
Steven H. Collins* \\ Dept. Mechanical Engineering \\ and Robotics Institute \\ Carnegie Mellon University \\ Pittsburgh, Pennsylvania 15213 \\ biomechatronics.cit.cmu.edu \\ stevecollins@cmu.edu
}

\author{
Peter G. Adamczyk \\ Intelligent Prosthetic Systems \\ Ann Arbor, Michigan 48103 \\ p.g.adamczyk@gmail.com
}

\begin{abstract}
The current process of prescribing prosthetic feet is hampered by imprecise classifications based on self-assessment, recommendations based on subjective prediction, burdensome justification requirements, and slow, costly testing of devices. These problems have been exacerbated by the introduction of robotic prostheses, which can improve gait performance for some individuals, but are very expensive. We propose an alternative process, in which a versatile robotic emulator is used to preview patient interactions with a range of prostheses, while objective data related to effort, stability, speed and preference are collected, all prior to prescription. Results from pilot testing with a prototype emulator system demonstrate accurate haptic rendering of a wide range of prosthesis classes and differentiation of user performance across these classes. Eventually, emulation-based prescription could reduce bias, cost and waste in the prescription process, while simultaneously improving patient outcomes.
\end{abstract}

\section{The Conventional Prescription Process}

About 150,000 lower-limb prosthesis prescriptions are made each year in the United States. The majority arise from the more than 100,000 lower-limb amputations performed each year, while others are the result of replacement, e.g., due to wear. During a typical prescription, the patient first receives a generic script from a physician. A prosthetist then recommends a particular device based on the patient's self-reported activity level, weight and height, and residual limb geometry. These data have low resolution, and the prosthetist's subjective judgment and personal experience therefore must play a significant role in the recommendation. Following verification from the physician, a prosthetic device is ordered and a custom socket adapter is fabricated for the patient's residual limb. Finally, the patient receives the complete prosthesis, and the prosthetist performs a fitting in which the device is iteratively adjusted during short bouts of standing and walking.

\section{A. Shortcomings of the conventional process}

Although conventional prescriptions seem to result in good outcomes in most cases, there are serious shortcomings. Selfreported data are subjective and imprecise, and cannot anticipate each individual's complex dynamical interactions with new mechanical elements attached to their body. Prosthetist recommendations are largely subjective, and can be biased by positive or negative experiences with a product as it was used by a previous patient. Once a device has been purchased, it is difficult to replace if the expected advantages are not realized. Prosthetists also have an economic incentive to recommend more expensive devices, which may have features that do not benefit a particular user. In response to instances of abuse, federal reimbursement standards have recently been changed to require more involvement of the prescribing physician, as they have less potential for conflict of interest. Insurance carriers now have high standards for documentation in cases where more advanced prosthetic limbs are prescribed.

These problems are exacerbated by the emergence of expensive, high-performance robotic prostheses. A typical prosthetic foot for individuals with low activity level, such as a solid ankle cushioned heel (SACH) foot, has a catalog price of less than $\$ 100$. A typical prosthesis for active individuals, such as the Össur Vari-Flex ${ }^{\circledR}$, has a catalog price of about $\$ 1,000$. New robotic prostheses for highly active individuals, such as the $\mathrm{iWalk} \mathrm{BiOM}^{\circledR}$, have a catalog price of about $\$ 80,000$. Incorrect prescriptions therefore cause order-of-magnitude differences in cost, driving economic inefficiency. On the other hand, advanced robotic prostheses substantially improve mobility for some users [1]. Hesitance on the part of a practitioner to prescribe such an expensive device, or to justify the purchase to an insurance company, can therefore inhibit mobility for more capable patients.

\section{PRESCRIPTION USING Robotic EMUlAtors}

We propose an alternative process, in which a versatile prosthesis 'emulator' system is used to preview patient interactions with candidate devices prior to prescription. The robotic emulator acts as a haptic interface, providing the same mechanical behavior as the emulated prosthesis, thereby allowing the patient to experience candidate devices virtually. Different prosthesis behaviors are pre-programmed into the system, and modes and parameters are switched online through a software interface. Many different designs can therefore be experienced in a short period of time, without time-consuming hardware changes and re-fittings, and without the need for an extensive physical inventory of candidate prostheses with each possible combination of features. 
A Emulator system

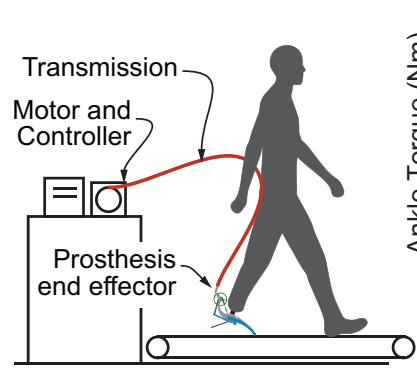

B SACH

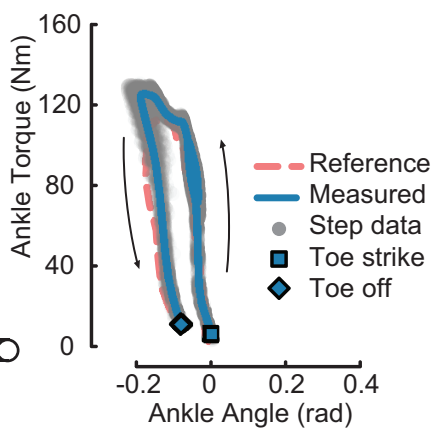

C DER

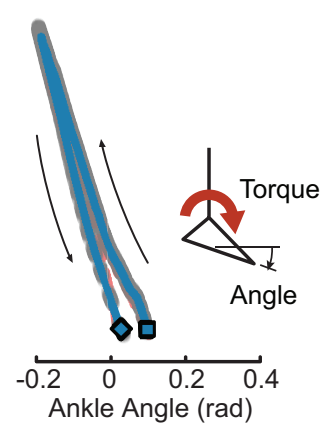

D BiOM

E HiPow

Fig. 1. Emulating commercial and hypothetical prostheses. A. The prosthesis emulator comprises powerful off-board motor and control hardware, a flexible tether, and a lightweight instrumented prosthesis, resulting in high responsiveness and low worn mass. The system has demonstrated high-quality haptic emulations of: B economical, but low-performance, solid-ankle cushioned heel (SACH) feet, which are stiff and dissipate significant energy, $\mathbf{C}$ mid-range dynamic-elastic response (DER) feet, which are more compliant and spring-like, D expensive, active robotic feet (BiOM), which use motors to generate positive work, and $\mathbf{E}$ conceptual designs, such as this high-powered robotic (HiPow) foot. Changing modes and tuning properties can be performed online without the need for time-consuming physical swaps or expansive inventories of test prostheses. Human response to these modes of operation can then be evaluated, for example based on indicators of effort, maximum speed, stability or preference. An appropriate prescription can then be made, with quantitative data automatically generated to justify the decision. Data in B-E are from a single individual with trans-femoral amputation over one hundred strides.

A robotic prosthesis emulator system can also collect objective data to guide recommendations and justify prescriptions. This includes measures of overall effort, such as heart rate, measures of gait stability, such as kinematic variability of foot placement, and measures of maximal performance, such as peak sustainable speed. These measures could augment or replace conventional K-level classifications, integer scores from 0 to 4 based on self-reported activity. Patients could also report preference among devices in an informed way. Prosthetists would have an opportunity to visually evaluate patient gait under different modes, and a video recording could be made for later inspection by the physician. Bias in the process could be mitigated by randomizing and anonymizing prosthesis modes. The result would be a rigorous set of objective data and unbiased subjective observations, which could facilitate better recommendations by the prosthetist and provide documentation for the prescribing physician.

\section{A. Prototype robotic ankle-foot prosthesis emulator}

We have designed and tested a prototype system capable of emulating existing prosthetic limbs as part of an improved prescription process. This tethered ankle-foot prosthesis emulator (Fig. 1A) is exceptionally versatile, having, e.g., higher closed-loop torque bandwidth than any comparable system [2].

\section{B. Initial emulation results}

This system has enabled high-fidelity emulation of commercial prostheses. Initial dynamic characterizations used configuration-based impedance matching (Fig. 1B-E) and achieved root-mean-squared torque tracking errors of $1 \%$ to $4 \%$ of peak torque. With recent improvements in torque control [3], we expect even more accurate device renderings in the future, including distinctions among fine details of prosthesis models. Two participants with transtibial amputation who used the device subjectively reported that the modes felt similar to physical prostheses from each class that they had worn previously.

\section{Initial justification data results}

In initial tests with two participants with transtibial amputation, each of whom was rated K3 and used a DER prosthesis, we found that modes resulted in different metabolic rate, maximum speed, and user preference. For example, participants experienced the lowest metabolic rate with the BiOM emulation, but preferred the DER emulation. Future tests among subjects with a range of gait capacities will determine feasibility of this approach in differentiating individuals who would benefit from more advanced prostheses from those who would not. Future tests with clinical practitioners will determine whether this data augments the prescription process.

\section{SOCIETAL IMPLiCATIONS}

Emulation-based prosthesis prescription could lower health care costs by avoiding over-prescription of expensive devices, reduce overhead in justifying purchases to insurance carriers, and improve quality of life for people with amputation by quickly identifying designs that maximize improvements in mobility and lead to greater satisfaction.

\section{ACKNOWLEDGMENTS}

This material is based upon work supported by the National Institutes of Health under Award No. 1R43HD076518-01.

[1] H. M. Herr and A. M. Grabowski, "Bionic ankle-foot prosthesis normalizes walking gait for persons with leg amputation," Proc. Roy. Soc. Lon. B, vol. 279, pp. 457464, 2012.

[2] J. M. Caputo and S. H. Collins, "A universal anklefoot prosthesis emulator for experiments during human locomotion," J. Biomech. Eng., vol. 136, p. 035002, 2014.

[3] J. Zhang, C. Cheah, and S. H. Collins, "A systematic comparison of prominent torque control methods in a tethered ankle exoskeleton with series elastic actuation during human walking," Int. J. Rob. Res., vol. submitted, 2014. 